\title{
Clinical characteristics, imaging phenotypes and events free survival in Takayasu arteritis patients with hypertension
}

\author{
Sun Ying ${ }^{1}$, Wu Sifan ${ }^{1}$, Wang Yujiao ${ }^{1}$, Chen Rongyi ${ }^{1}$, Huang Qingrong ${ }^{1}$, Ma Lili ${ }^{1}, 2$, Chen Huiyong ${ }^{1}$ and Jiang Lindi ${ }^{1,2^{*}}$
}

\begin{abstract}
Background: Hypertension occurred in 30-80\% of Takayasu arteritis (TAK) patients around the world and the occurrence of hypertension might worsen the disease prognosis. This study aimed to investigate the clinical characteristics and imaging phenotypes, as well as their associations with events free survival (EFS) in Chinese TAK patients with hypertension.

Methods: This current research was based on a prospectively ongoing observational cohort-the East China Takayasu Arteritis (ECTA) cohort, centered in Zhongshan Hospital, Fudan University. Totally, 204 TAK patients with hypertension were enrolled between January 2013 and December 2019. Clinical characteristics and imaging phenotypes of each case were evaluated and their associations with the EFS by the end of August 30, 2020, were analyzed.
\end{abstract}

Results: Severe hypertension accounted for $46.1 \%$ of the entire population. Three specific imaging phenotypes were identified: Cluster 1: involvement of the abdominal aorta and/or renal artery (27.5\%); Cluster 2: involvement of the ascending aorta, thoracic aorta, the aortic arch, and/or its branches (18.6\%); and Cluster 3: combined involvement of Cluster 1 and 2 (53.9\%). Clinical characteristics, especially hypertensive severity, differed greatly among the three imaging clusters. In all, 187 patients were followed up for a median of 46 (9-102) months; 72 events were observed in 60 patients (1-3 per person). The overall blood pressure control rate was 50.8\%, and the EFS was $67.9 \%$ by the end of the follow-up. Multivariate Cox regression indicated that controlled blood pressure $(\mathrm{HR}=2.13,95 \% \mathrm{Cl} 1.32-3.74)$, Cluster $1(\mathrm{HR}=0.69,95 \% \mathrm{Cl} 0.48-0.92)$ and Cluster $3(\mathrm{HR}=0.72,95 \% \mathrm{Cl} 0.43-0.94)$ imaging phenotype was associated with the EFS. Kaplan-Meier curves showed that patients with controlled blood pressure showed better EFS $(p=0.043)$. Furthermore, using cases with Cluster 1 imaging phenotype and controlled blood pressure as reference, better EFS was observed in patients with Cluster 2 phenotype and controlled blood pressure ( $H R=2.21,95 \% \mathrm{Cl} 1.47-4.32)$, while the case with Cluster 1 phenotype plus uncontrolled blood pressure $(H R=0.64,95 \% \mathrm{Cl} 0.52-0.89)$ and those with Cluster 3 phenotype and uncontrolled blood pressure $(H R=0.83$, $95 \% \mathrm{Cl}$ 0.76-0.92) suffered worse EFS.

Conclusion: Blood pressure control status and imaging phenotypes showed significant effects on the EFS for TAK patients with hypertension.

\footnotetext{
* Correspondence: zsh-rheum@hotmail.com

'Department of Rheumatology, Zhongshan Hospital, Fudan University,

Shanghai, People's Republic of China

${ }^{2}$ Centre of Evidence-based Medicine, Zhongshan Hospital, Fudan University,

Shanghai, People's Republic of China
}

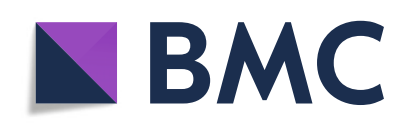

(c) The Author(s). 2021 Open Access This article is licensed under a Creative Commons Attribution 4.0 International License, which permits use, sharing, adaptation, distribution and reproduction in any medium or format, as long as you give appropriate credit to the original author(s) and the source, provide a link to the Creative Commons licence, and indicate if changes were made. The images or other third party material in this article are included in the article's Creative Commons licence, unless indicated otherwise in a credit line to the material. If material is not included in the article's Creative Commons licence and your intended use is not permitted by statutory regulation or exceeds the permitted use, you will need to obtain permission directly from the copyright holder. To view a copy of this licence, visit http://creativecommons.org/licenses/by/4.0/. The Creative Commons Public Domain Dedication waiver (http://creativecommons.org/publicdomain/zero/1.0/) applies to the data made available in this article, unless otherwise stated in a credit line to the data. 
Keywords: Takayasu arteritis, Hypertension, Hypertensive severity, Imaging phenotype, Blood pressure control status, Events free survival

\section{Background}

Takayasu arteritis (TAK) is a chronic inflammatory large-vessel vasculitis that primarily affects the aorta and its main branches [1-3]. Hypertension is a particularly important complication in patients with TAK [4-6]. According to previous reports, hypertension occurred in $33-83 \%$ of patients with TAK from different areas of the world, with younger age of disease onset [7-10]. The occurrence of hypertension could severely worsen TAK prognosis and may be a significant prognostic predictor of outcomes [11]. Furthermore, uncontrolled blood pressure was associated with a higher 5 years all-cause mortality risk than that in the healthy population, despite effective control of disease activity $[12,13]$. Thus, comprehensive understanding of the disease characteristics of TAK patients with hypertension is very essential.

To our knowledge, few studies have focused on hypertension in the TAK population up to date. In a previous research, we reported that in patients with TAK-related renal artery stenosis, the prevalence of hypertension was up to $60 \%$, with $30 \%$ refractory hypertensive cases [14]. Except renal artery stenosis, the involvement of abdominal aorta, as well as severe aortic regurgitation (AR) also could cause hypertension in TAK [15-17]. Nevertheless, data describing whether there are specific imaging features in TAK patients with hypertension as well as their associations with disease prognosis were still lacking.

Thus, this study was designed to investigate the clinical characteristics and specific imaging phenotypes of TAK patients with hypertension and to point out the associations of the clinical characteristics and imaging phenotypes with the events free survival (EFS).

\section{Methods}

\section{Study design and subjects}

The present study was based on a prospectively ongoing observational cohort-the East China Takayasu Arteritis (ECTA) cohort, centered in Zhongshan Hospital, Fudan University, Shanghai, China. All patients enrolled into the ECTA cohort had a confirmed diagnosis of TAK based on the 1990 American College of Rheumatology (ACR) criteria [18]. The demographic, clinical, laboratory, and treatment data were collected at baseline and each visit. The follow-up frequency was once a month in the active phase and once every 3 months in the remission phase. Disease activity was assessed using the National Institutes of Health (NIH) criteria [19]. The clinical data of all enrolled patients were recorded and stored in a unified electronic database (REDCap database system, https://redcap.zs-hospital.sh.cn).

In all, 204 TAK patients with hypertension, were enrolled in to the current research from the ECTA cohort between January 2013 and December 2019. Clinical characteristics and imaging features of each case were evaluated by professional rheumatologists. The main outcome of the investigation was the EFS by the end of August 30, 2020 (Fig. 1). Associations of the clinical and imaging features with the EFS were analyzed. The study was performed in accordance with the tenets of the Helsinki Declaration and its amendments. The study protocol was approved by the Ethics Review Board of Zhongshan Hospital (B2013-115(3)). Written informed consent was obtained from all patients.

\section{Blood pressure measurement}

Upper-limb blood pressure was measured in all patients mainly at home. Blood pressure measurement of the four limbs and ankle brachial index (ABI) was performed in $92 / 204(45.1 \%)$ patients at clinic by using a noninvasive blood pressure monitor (BP-203RPEIII, Omron Healthcare Co., Ltd., Tokyo, Japan). For those without subclavian artery involvement, the reading from the arm with the higher value was used as the reference measurement. For patients with unilateral subclavian artery involvement, the reading from the unaffected side was analyzed, while for those with bilateral subclavian artery involvement, higher values of blood pressure of the lower limbs were used for analysis.

\section{Classification of hypertensive severity and blood pressure control status}

The severity of hypertension was classified at baseline as previously reported [20]: (i) mild: a brachial pressure of $140-159 \mathrm{mmHg}$ systolic or $90-99 \mathrm{mmHg}$ diastolic; (ii) moderate: a brachial pressure of $160-179 \mathrm{mmHg}$ systolic or $100-109 \mathrm{mmHg}$ diastolic; and (iii) severe: a brachial pressure of $\geq 180 \mathrm{mmHg}$ systolic or $\geq 110 \mathrm{mmHg}$ diastolic. The blood pressure should satisfy the standard in at least 20 days of a month.

Blood pressure control status was classified as: (i) controlled: systolic blood pressure (SBP) $<140 \mathrm{mmHg}$ and diastolic blood pressure (DBP) $<90 \mathrm{mmHg}$; (ii) improved: $\mathrm{SBP} \geq 140 \mathrm{mmHg}$, but decreased by $\geq 20 \mathrm{mmHg}$ and/or DBP $\geq 90 \mathrm{mmHg}$, but decreased by $\geq 10 \mathrm{mmHg}$; and (iii) failed: failed meeting the above-defined criteria [21]. The blood pressure should achieve the relative standard in 20 days per month for at least 3 months. 


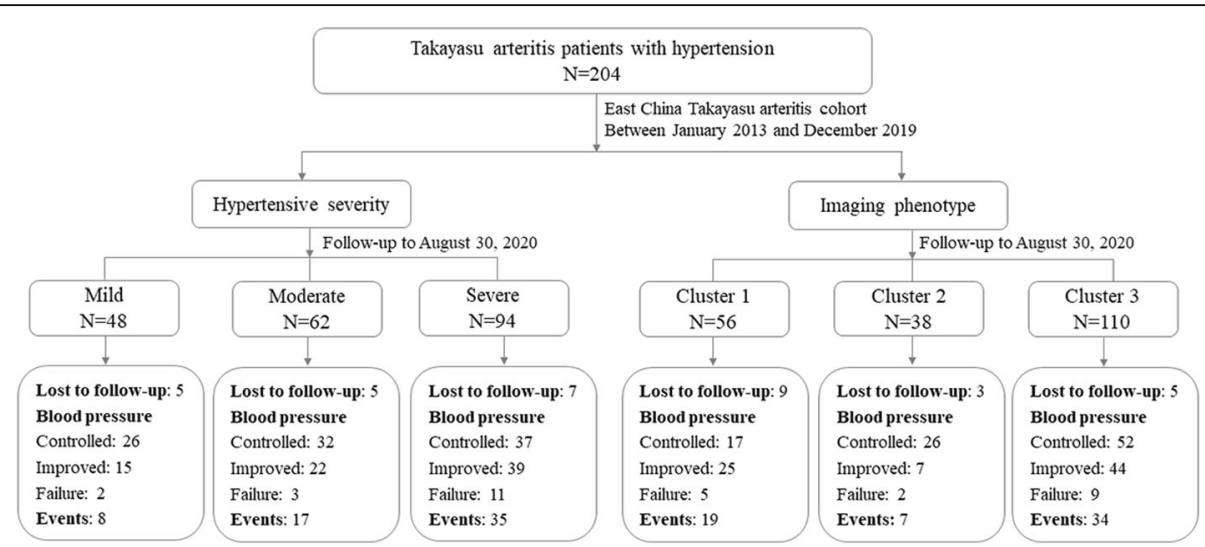

Fig. 1 Study flow chart. In all, 204 hypertensive Takayasu arteritis patients were enrolled in to the present study from the East China Takayasu arteritis cohort between January 2013 and December 2019. Clinical characteristics and imaging features of each case were evaluated. The main outcome of the investigation was the events free survival by the end of August 30, 2020. Subgroup analysis, according to hypertensive severity and imaging phenotype, was also performed. Cluster 1: involvement of abdominal aorta and/or renal artery; Cluster 2: involvement of ascending aorta, thoracic aorta, and/or the aortic arch and its branches; and Cluster 3: combined involvement of Cluster 1 and Cluster 2.

Refractory hypertension was defined as a brachial pressure $\geq 160 \mathrm{mmHg}$ systolic or $\geq 90 \mathrm{mmHg}$ diastolic pressure despite maximal doses of three antihypertensive drugs for at least 1 month [20,21].

\section{Imaging measurements}

Imaging assessments, mainly the whole-body enhanced magnetic resonance angiography (MRA) or computed tomography angiography (CTA), were performed at the time of enrollment. Angiography findings were classified according to the classification by Numano et al. in 1996 [22].

Diagnosis of AR was confirmed by echocardiography according to the guideline of the American Society of Echocardiography [23]. The severity of AR was evaluated by echocardiography as previously described $[24,25]$.

\section{Outcomes}

Patients, who completed at least 6 months of follow-up, were included in the outcome analysis. The occurrence of any events during the follow-up included (i) renal insufficiency including new occurrence, persistent insufficiency ( $\geq 6$ months) or deterioration of renal function $(\geq$ $20 \%$ increase in creatinine concentration or $\geq 20 \%$ decrease in glomerular filtration rate (GFR)); (ii) persistent refractory hypertension ( $\geq 6$ months) or malignant hypertension; (iii) congestive heart failure including new occurrence or deterioration of heart function; (iv) new occurrence of cerebrovascular events; (v) arterial dissection or rupture of aneurysms; or (vi) TAK-related death (e.g., death caused by severe arterial stenosis or aortic dissection).

\section{Statistical analysis}

Categorical variables were summarized as counts and percentages and were compared using chi-square or
Kruskal-Wallis tests. Continuous variables are presented as means \pm standard deviations (SD) or as medians with interquartile range (IQR), depending on the normality of distribution, and were compared using Student's $t$ tests, Wilcoxon tests, or one-way ANOVA. For variables with significant differences among three or more groups, pair-wise comparisons were further performed using Student's $t$ tests, Wilcoxon tests, or chi-square tests.

To identify specific imaging phenotypes for hypertensive TAK patients, 14 arteries including bilateral carotid arteries, brachiocephalic trunk, bilateral subclavian arteries, aortic arch, ascending aorta, thoracic aorta, pulmonary artery, abdominal aorta, bilateral renal artery, superior mesenteric artery, and celiac axis were included in the cluster analysis by a two-step progress as described previously [26]. Tree dendograms were created to visualize cluster patterns.

Cox proportional hazards regression model was used to evaluate associations of imaging phenotypes and clinical characteristics with EFS during the follow-up by adjusting for age, sex, disease duration, disease activity, and received treatment including medications and revascularization procedures. Hazard ratios (HR) and 95\% confidence intervals (CIs) were reported. The KaplanMeier method was used to plot the proportion distribution of EFS in the above subgroups over time with logrank test. Statistical analyses were performed using SPSS 22.0 (IBM Corp., Armonk, NY, USA). Two-sided $p<$ 0.05 was considered to indicate statistical significance.

\section{Results}

\section{Patients general characteristics}

In total, 204 (33\%) patients (155 [76\%] female) suffered from hypertension in our cohort, with dizziness/headache $(88 / 204,43.1 \%)$, chest distress $(57 / 204,27.9 \%)$, and 
weakness $(58 / 204,28.4 \%)$ as the most common manifestations. According to the 1996 classification, type V (112/204, 54.9\%) was the most common imaging type, followed by type IV (44/204, 21.6\%). The demographic and clinical characteristics were presented in Supplementary Table S1.

In comparison with patients without hypertension, a higher prevalence of renal insufficiency $(8.8 \%$ vs. $2.2 \%, p$ $=0.001)$ and heart failure $(11.8 \%$ vs. $5.8 \%, p=0.009)$ was observed in patients with hypertension. Imaging types and arterial involvement significantly differed $(p<$ 0.001 ), showing a higher prevalence of renal artery involvement $(56.9 \%$ vs. $13.3 \%, p<0.001)$ and abdominal aorta involvement $(51.5 \%$ vs. $22.7 \%, p<0.001)$ in patients with hypertension (Supplementary Table S1).

\section{Characteristics of patients with different hypertensive severity}

Mild, moderate, and severe hypertension was observed in $48(23.5 \%), 62(30.4 \%)$, and $94(46.1 \%)$ cases, respectively. Clinical characteristics of the three categories were summarized in Table 1. Age, sex, and disease duration were similar among the three categories. The prevalence of renal insufficiency $(p=0.048)$, renal artery involvement $(p=0.043)$, as well as blood pressure control status $(p<0.001)$ significantly differed among the three hypertension categories. Patients with severe hypertension were more likely to experience failure control of blood pressure than those with mild ( $12.6 \%$ vs. $4.6 \%, p$ $<0.001)$ and moderate hypertension $(12.6 \%$ vs. $5.3 \%, p=$ $0.008)$, respectively.

Immunosuppressive therapy including the oral dose of glucocorticoids and the choice of immunosuppressors did not significantly differ the three hypertensive categories, while the medium kind of antihypertensive drugs and the medication choice differed. Patients with severe hypertension had higher revascularization rates than those with moderate hypertension $(45.7 \%$ vs. $24.2 \%$, $p=0.008$ ) (Supplementary Table S2).

\section{Characteristics of patients with different imaging phenotypes}

As significant differences of artery involvement were demonstrated in patients with hypertension, cluster analysis was further performed to explore new imaging phenotypes for TAK patients with hypertension (Supplementary Fig S1): Cluster 1: involvement of abdominal aorta and/or renal artery $(n=56,27.5 \%)$; Cluster 2: involvement of ascending aorta, thoracic aorta, and/or the aortic arch and its branches $(n=38,18.6 \%)$; and Cluster 3: combined involvement of Cluster 1 and Cluster $2(n=110,53.9 \%)$. The clinical characteristics of patients with different imaging phenotypes were shown in Table 2.
Besides sex $(p=0.007)$ and age $(p<0.001)$, the prevalence of baseline features, including renal insufficiency $(p=0.046)$, heart failure $(p=0.013)$, cerebral infarction $(p=0.007)$, severe hypertension $(p=0.014)$, and severe $\mathrm{AR}(p=0.047)$ differed significantly among the three imaging phenotype clusters. Furthermore, Cluster 1 had a higher prevalence of severe $(>75 \%)$ renal artery stenosis than Cluster 3 ( $51.8 \%$ vs. $36.4 \%, p=0.036)$, though Cluster 3 involves some same lesions with Cluster 1 . The blood pressure control status also differed among the three clusters of imaging phenotypes. Patients with Cluster 1 imaging phenotype experienced a lower prevalence of controlled hypertension $(36.2 \%$ vs. $74.3 \%, p<0.001)$ and higher prevalence of failure control $(10.6 \%$ vs. $5.7 \%$, $p=0.019$ ) than those with Cluster 2 phenotype (Fig. 2). In addition, immunosuppressive and anti-hypertensive therapy did not significantly differ among patients with different imaging phenotypes (Supplementary Table S3).

\section{Characteristics of patients with different blood pressure control status}

The overall blood pressure controlled, improved, and failure rates were $50.8 \%, 40.6 \%$, and $8.6 \%$, respectively. The clinical characteristics of patients with different blood pressure control status were summarized in Table 3 . The prevalence of severe AR differed among patients with different blood pressure control status $(p<0.001)$. Lower prevalence of Cluster 1 imaging phenotype was observed in the controlled group compared with improved $(17.9 \%$ vs. $32.9 \%, p=0.031)$ and failure group ( $7.9 \%$ vs. $31.3 \%, p=0.047)$. Meanwhile, higher prevalence of Cluster 2 imaging phenotype was also observed in the controlled group compared with improved $(27.4 \%$ vs. $9.2 \%, p=0.001)$ and failure group $(27.4 \%$ vs. $12.5 \%, p$ $=0.024$ ) (Fig. 3).

What is more, immunosuppressive treatments did not significantly differ among patients with different blood pressure control status, while anti-hypertensive treatment differed greatly. Patients in the controlled group showed more usage of angiotensin-converting enzyme inhibitor/angiotensin receptor blocker (ACEI/ARB) $(33.7 \%$ vs. $12.5 \%, p<0.001)$ and $\beta$-blocker $(64.2 \%$ vs. $31.3 \%, p=0.022)$ compared with the failure group (Fig. 3).

\section{Events free survival by the end of the follow-up}

Totally, 187/204 (91.7\%) patients were followed up for a median of 46 (9-102) months, among whom, 127 cases did not experience any events, with a medium follow-up duration of $36(8-100)$ months. Seventy-two events (1-3 per person) were observed in $60(32.1 \%)$ patients, with a median follow-up duration of 48 (6-92) months. The events included persistent refractory or malignant hypertension $(27,14.4 \%)$, persistent or deteriorated renal 
Table 1 Characteristics of patients with different hypertensive severity

\begin{tabular}{|c|c|c|c|c|}
\hline & Mild & Moderate & Severe & $P$-value \\
\hline Baseline assessment & $N=48$ & $N=62$ & $N=94$ & \\
\hline \multicolumn{5}{|l|}{ Demography } \\
\hline Female $(n, \%)$ & $35(72.9 \%)$ & $47(75.8 \%)$ & $71(75.5 \%)$ & 0.752 \\
\hline Age (years, IQR) & $40(27-49)$ & $35(27-46)$ & $37(24-48)$ & 0.271 \\
\hline Disease duration (months, IQR) & $24(2-96)$ & $34(6-96)$ & $24(3-102)$ & 0.513 \\
\hline \multicolumn{5}{|l|}{ Clinical manifestation ( $n, \%)$} \\
\hline Systemic symptoms (fever, weakness, etc.) & $19(39.6 \%)$ & $24(38.7 \%)$ & $34(36.2 \%)$ & 0.924 \\
\hline Neurological symptoms (headache, amaurosis, etc.) & $14(29.2 \%)$ & $26(41.9 \%)$ & $55(58.5 \%)$ & 0.544 \\
\hline Cardiovascular symptoms (chest distress/pain, etc.) & $9(18.8 \%)$ & $16(25.8 \%)$ & $32(34.1 \%)$ & 0.273 \\
\hline \multicolumn{5}{|l|}{ Complications ( $n, \%)$} \\
\hline Renal insufficiency & $2(4.2 \%))^{\#, *}$ & $5(8.1 \%)$ & $11(11.7 \%)$ & 0.048 \\
\hline Heart failure & $4(8.3 \%)$ & $7(11.3 \%)$ & $13(13.8 \%)$ & 0.701 \\
\hline Cerebral infarction & $2(4.2 \%)$ & $2(3.2 \%)$ & $5(5.3 \%)$ & 0.821 \\
\hline \multicolumn{5}{|l|}{ Artery involvement $(n, \%)$} \\
\hline Abdominal aorta & $20(41.7 \%)$ & $32(51.6 \%)$ & $53(56.4 \%)$ & 0.293 \\
\hline Renal artery & $20(41.7 \%)^{*}$ & $28(45.2 \%)^{8}$ & $68(72.3 \%)$ & 0.043 \\
\hline Thoracic aorta & $10(20.8 \%)$ & $22(35.5 \%)$ & $32(34.1 \%)$ & 0.293 \\
\hline Carotid artery & $13(27.1 \%)$ & $26(41.9 \%)$ & $39(41.5 \%)$ & 0.115 \\
\hline Subclavian artery & $27(52.1 \%)$ & $33(53.2 \%)$ & $47(50.0 \%)$ & 0.744 \\
\hline \multicolumn{5}{|l|}{ Echocardiography ( $n, \%)$} \\
\hline Severe AR & $3(6.3 \%)$ & $5(8.1 \%)$ & $11(11.7 \%)$ & 0.082 \\
\hline Follow-up assessment & $N=43$ & $N=57$ & $N=87$ & \\
\hline \multicolumn{5}{|l|}{ Blood pressure control status ( $n, \%)$} \\
\hline Controlled & $26(60.5 \%)$ & $32(56.1 \%)$ & $37(42.5 \%)$ & 0.082 \\
\hline Improved & $15(34.9 \%)$ & $22(38.6 \%)$ & $39(44.8 \%)$ & 0.727 \\
\hline Failure & $2(4.6 \%)^{*}$ & $3(5.3 \%)^{\&}$ & $11(12.6 \%)$ & $<0.001$ \\
\hline \multicolumn{5}{|l|}{ Events $(n, \%)$} \\
\hline Persistent refractory or malignant hypertension & $3(6.9 \%)^{\#, *}$ & $8(14.0 \%)$ & $16(18.4 \%)$ & 0.016 \\
\hline Renal insufficiency & $2(4.7 \%)$ & $4(7.0 \%)$ & $6(6.9 \%)$ & 0.322 \\
\hline Congestive heart failure & $2(4.7 \%)$ & $3(5.3 \%)$ & $2(2.3 \%)$ & 0.069 \\
\hline Events free survival by the end & $81.4 \% *$ & $70.2 \%$ & $59.8 \%$ & 0.047 \\
\hline
\end{tabular}

$A R$ aortic regurgitation; $P$ value: comparison among patients with different hypertensive severity; ${ }^{\#} p<0.05$ for comparisons between patients with mild and moderate hypertension; ${ }^{*} p<0.05$ for comparisons between patients with mild and severe hypertension; ${ }^{\circledR} p<0.05$ for comparisons between patients with between patients with moderate and severe hypertension

insufficiency $(12,6.4 \%)$, heart dysfunction $(7,3.7 \%)$, cerebrovascular events $(5,2.7 \%)$, aortic dissection $(2,1.1 \%)$, abdominal aortic dissection (2,1.1\%), rupture of abdominal aortic aneurysm (1, 0.5\%), and death (4, 2.1\%: two due to heart dysfunction, one due to rupture of aortic dissection, and one due to cerebrovascular event after bypass surgery of thoracic and abdominal aorta).

The EFS by the end of the follow-up was $67.9 \%$ in the entire population, while it was $81.4 \%, 70.8 \%$, and $59.8 \%$ in patients with mild, moderate, and severe hypertension, respectively; $59.6 \%, 80.0 \%$, and $67.6 \%$ in patients with Cluster 1, Cluster 2, and Cluster 3 imaging phenotype, respectively; and $75.8 \%, 61.8 \%$, and $50.0 \%$ in patients with controlled, improved, and control failure hypertension, respectively (Tables 1 and 2).

\section{Associations of the clinical characteristics and imaging phenotypes with events free survival}

Multivariate Cox regression analysis, with adjustments of age, sex, disease duration, disease activity, and received medications, indicated that co-existence of severe AR $(\mathrm{HR}=0.87,95 \% \mathrm{CI} 0.64-0.95, p=0.043)$, controlled blood pressure $(\mathrm{HR}=2.13,95 \% \mathrm{CI} 1.32-3.78, p=0.047)$, Cluster $1(\mathrm{HR}=0.69,95 \% \mathrm{CI} 0.48-0.92, p=0.017)$, and 
Table 2 Characteristics of patients with different imaging phenotypes

\begin{tabular}{|c|c|c|c|c|}
\hline & Cluster 1 & Cluster 2 & Cluster 3 & $P$-value \\
\hline Baseline assessment & $N=56$ & $N=38$ & $N=110$ & \\
\hline \multicolumn{5}{|l|}{ Demography } \\
\hline Female $(n, \%)$ & $34(59.6 \%)^{*}$ & $30(78.9 \%)$ & $91(82.7 \%)$ & 0.007 \\
\hline Age (years, IQR) & $27(20-39)^{\#}$ & $42(32-54)$ & $39(27-49)$ & $<0.001$ \\
\hline Disease duration (months, IQR) & $12(3-56)$ & $33(4-72)$ & $30(4-120)$ & 0.334 \\
\hline \multicolumn{5}{|l|}{ Clinical manifestation $(n, \%)$} \\
\hline Systemic symptoms (fever, weakness, etc.) & $15(26.8 \%)$ & $17(44.7 \%)$ & $45(40.9 \%)$ & 0.320 \\
\hline Neurological symptoms (headache, amaurosis, etc.) & $17(30.3 \%)$ & $21(55.3 \%)$ & $51(46.4 \%)$ & 0.461 \\
\hline Cardiovascular symptoms (chest distress/pain, etc.) & $10(17.9 \%)^{\#}$ & $16(42.1 \%)$ & $31(28.2 \%)$ & 0.010 \\
\hline \multicolumn{5}{|l|}{ Complications ( $n, \%)$} \\
\hline Renal insufficiency & $5(8.9 \%)^{\#}$ & $1(2.6 \%)^{\&}$ & $12(10.9 \%)$ & 0.046 \\
\hline Heart failure & $1(1.8 \%)^{\#, *}$ & $5(13.2 \%)$ & $18(16.4 \%)$ & 0.013 \\
\hline Cerebral infarction & $1(1.8 \%)^{*}$ & 0 & $8(7.3 \%)$ & 0.007 \\
\hline \multicolumn{5}{|l|}{ Hypertensive severity $(n, \%)$} \\
\hline Moderate & $18(32.1 \%)$ & $12(31.6 \%)$ & $32(29.1 \%)$ & 0.217 \\
\hline Severe & $26(46.4 \%)$ & $10(26.3 \%)^{\&}$ & $58(52.7 \%)$ & 0.014 \\
\hline \multicolumn{5}{|l|}{ Artery involvement $(n, \%)$} \\
\hline Renal artery stenosis $>75 \%$ & $29(51.8 \%)^{*}$ & 0 & $40(36.4 \%)$ & $<0.001$ \\
\hline Abdominal aorta stenosis > 50\% & 19 (33.9\%) & 0 & $24(21.8 \%)$ & $<0.001$ \\
\hline \multicolumn{5}{|l|}{ Echocardiography ( $n, \%)$} \\
\hline Severe AR & $4(7.1 \%)^{\#}$ & $5(11.4 \%)$ & $10(9.1 \%)$ & 0.047 \\
\hline Follow-up assessment & $N=47$ & $N=35$ & $N=105$ & \\
\hline \multicolumn{5}{|l|}{ Blood pressure control status $(n, \%)$} \\
\hline Controlled & $17(36.2 \%)^{\#}$ & $26(74.3 \%)$ & $52(47.3 \%)$ & $<0.001$ \\
\hline Improved & $25(53.2 \%)$ & $7(20.0 \%)$ & $44(40.0 \%)$ & 0.117 \\
\hline Failure & $5(10.6 \%)^{\#}$ & $2(5.7 \%)$ & $9(8.2 \%)$ & 0.032 \\
\hline \multicolumn{5}{|l|}{ Events $(n, \%)$} \\
\hline Persistent refractory or malignant hypertension & $9(19.1 \%)^{\#}$ & $3(8.6 \%)^{\&}$ & $15(13.6 \%)$ & 0.024 \\
\hline Renal insufficiency & $4(8.5 \%)$ & 0 & $8(7.3 \%)$ & 0.178 \\
\hline Congestive heart failure & $1(2.1 \%)^{\#}$ & $2(5.7 \%)^{\&}$ & $4(3.8 \%)$ & 0.017 \\
\hline Events free survival by the end & $59.6 \%{ }^{\#}$ & $80.0 \%$ & $67.6 \%$ & 0.049 \\
\hline
\end{tabular}

Cluster 1: involvement of abdominal aorta and/or renal artery; Cluster 2: involvement of ascending aorta, thoracic aorta, aortic arch and its branches; Cluster 3: combined involvement of Cluster 1 and Cluster 2; $A R$ aortic regurgitation; $P$ value: comparison among patients with different imaging phenotypes; ${ }^{\prime} p<0.05$ for comparisons between patients with Cluster 1 and Cluster 2 phenotype; ${ }^{*} p<0.05$ for comparisons between patients with Cluster 1 and Cluster 3 phenotype; and $\&_{p}<0.05$ for comparisons between patients with Cluster 2 and Cluster 3 phenotype.

Cluster 3 imaging phenotype $(\mathrm{HR}=0.72,95 \% \mathrm{CI}$ $0.43-0.94, p=0.048$ ) were significantly associated with the EFS (Table 4). In further analysis, patients, who had Cluster 1 imaging phenotype with controlled blood pressure was set as reference, cases with Cluster 2 imaging phenotype and controlled blood pressure showed better EFS (HR $=2.21,95 \%$ CI 1.47-4.32, $p=0.027$, while those had Cluster 1 imaging phenotype with uncontrolled blood pressure $(\mathrm{HR}=0.64$, 95\%CI 0.52-0.89, $p=0.031$ ) and Cluster 3 imaging phenotype with uncontrolled blood pressure (HR = $0.83,95 \%$ CI $0.76-0.92, p=0.048$ ) suffered worse EFS (Table 4).
Kaplan-Meier curves showed that patients with Cluster 1 imaging phenotype might suffer from worse EFS in comparison with Cluster 2 and Cluster 3 imaging phenotype, though this difference was not statistically significant (Fig. 4A). Patients with controlled blood pressure showed better EFS during the follow-up (Fig. 4B).

\section{Discussion}

The present study aimed to summarize the disease characteristics of TAK patients with hypertension and highlight potential determinants to the EFS. We found that (i) about $33 \%$ of TAK patients in our cohort suffered from hypertension, among whom, almost half were 


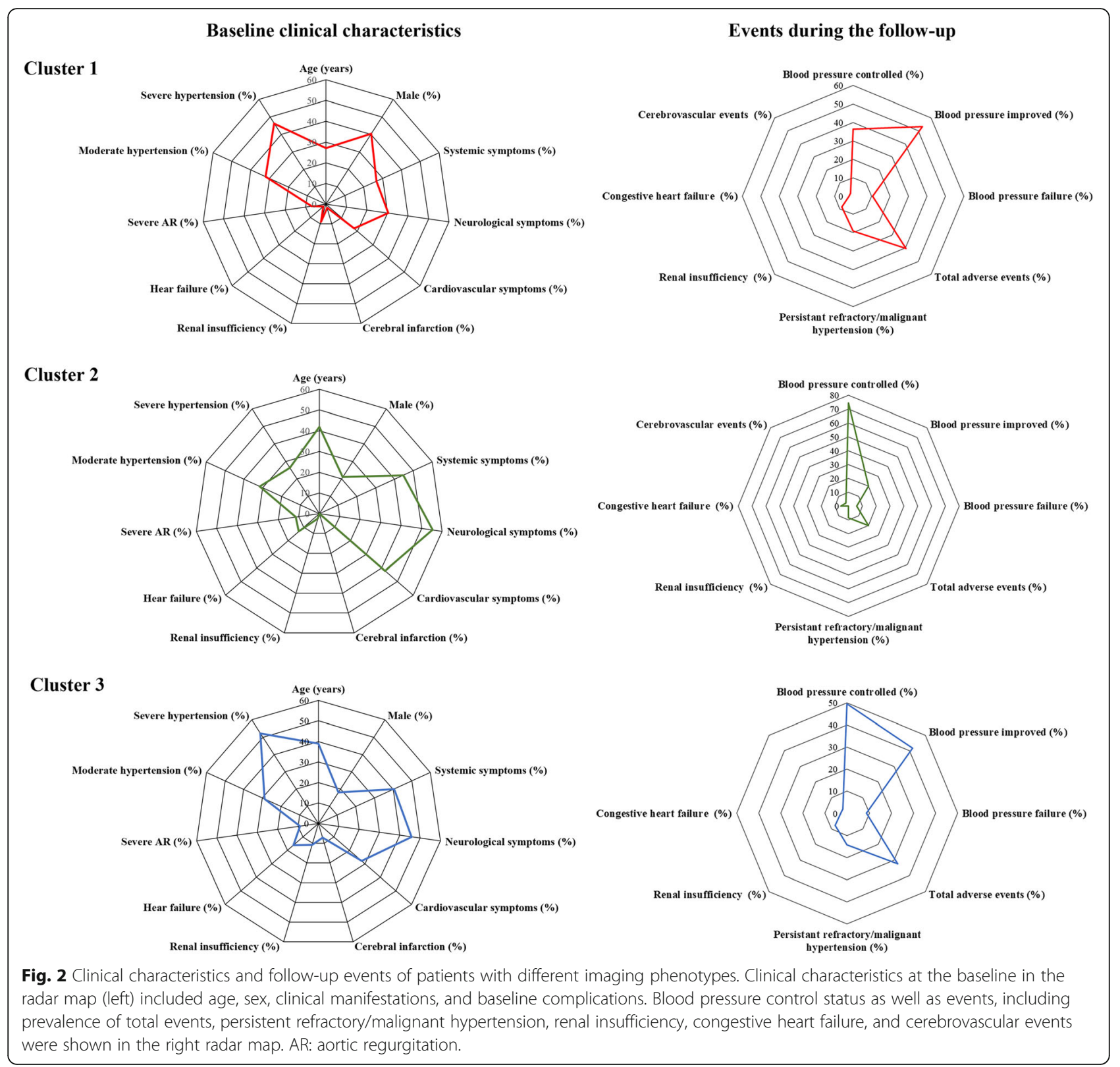

severe hypertension; (ii) three specific imaging phenotypes were identified for TAK patients with hypertension, which could be distinguished from those without hypertension; (iii) only $50.8 \%$ patients got controlled blood pressure in the present study and the overall EFS was $67.9 \%$ by the end of a median 48 months follow-up; and (iv) patients with controlled hypertension showed better EFS, while imaging phenotype also showed effects on the EFS, though not statistically significant.

Previous studies have reported that hypertension occurred in $33-83 \%$ of TAK patients, with younger disease onset age (mostly < 40 years) [7-10, 21]. One former study even indicated that a combination of hypertension and elevated erythrocyte sedimentation rate (ESR) was useful for diagnosing TAK in patients $<18$ years of age [27]. Our data pointed out that $33 \%$ of TAK patients suffered from hypertension, which was consistent with these previous studies. Furthermore, severe hypertension was observed in almost half of the hypertensive cases in our cohort, and severe hypertensive patients were more likely to complain of renal insufficiency and failure to control the elevated blood pressure. These findings call for physicians' awareness of the diagnosis of TAK in young individuals presenting with hypertension, especially in those with indecipherable severe hypertension.

Previous studies have revealed that renal artery stenosis-associated hypertension was observed in about $50 \%$ of TAK cases $[12,27,28]$. In the current study, we 
Table 3 Characteristics of patients with different blood pressure control status

\begin{tabular}{|c|c|c|c|c|}
\hline & $\begin{array}{l}\text { Controlled } \\
N=95\end{array}$ & $\begin{array}{l}\text { Improved } \\
N=76\end{array}$ & $\begin{array}{l}\text { Failure } \\
N=16\end{array}$ & $P$-value \\
\hline \multicolumn{5}{|l|}{ Demography } \\
\hline Female $(n, \%)$ & $72(75.8 \%)$ & $54(71.1 \%)$ & $12(75.0 \%)$ & 0.313 \\
\hline Age (years, IQR) & $31(24-42)$ & $34(22-39)$ & $29(21-38)$ & 0.411 \\
\hline \multicolumn{5}{|l|}{ Hypertensive severity ( $n, \%)$} \\
\hline Moderate & $34(35.8 \%)$ & 17 (22.4\%) & $5(37.5 \%)$ & 0.287 \\
\hline Severe & $44(45.3 \%)$ & $33(46.1 \%)$ & $10(56.3 \%)$ & 0.114 \\
\hline \multicolumn{5}{|l|}{ Echocardiography ( $n, \%)$} \\
\hline Severe AR & $9(9.5 \%)^{*}$ & $6(7.9 \%)^{8}$ & $4(25.0 \%)$ & $<0.001$ \\
\hline \multicolumn{5}{|l|}{ Imaging phenotype ( $n, \%)$} \\
\hline Cluster 1 & $17(17.9 \%))^{\#, *}$ & $25(32.9 \%)$ & $5(31.3 \%)$ & 0.035 \\
\hline Cluster 2 & $26(27.4 \%)^{\#, *}$ & $7(9.2 \%)$ & $2(12.5 \%)$ & $<0.001$ \\
\hline Cluster 3 & $52(54.7 \%)$ & $44(57.9 \%)$ & $9(56.3 \%)$ & 0.301 \\
\hline \multicolumn{5}{|l|}{ Immunosuppressive treatment } \\
\hline Glucocorticoid (prednisone, mg/day, IQR) & $35(15-40)$ & $30(7-40)$ & $30(15-40)$ & 0.317 \\
\hline Cyclophosphamide $(n, \%)$ & $20(21.2 \%)$ & $13(17.1 \%)$ & $5(31.3 \%)$ & 0.289 \\
\hline Leflunomide $(n, \%)$ & $21(22.1 \%)$ & $14(18.4 \%)$ & $4(25.0 \%)$ & 0.376 \\
\hline Biological agents $(n, \%)$ & $11(11.6 \%)$ & $4(5.3 \%)$ & $2(12.5 \%)$ & 0.053 \\
\hline \multicolumn{5}{|l|}{ Anti-hypertensive treatment } \\
\hline Number of antihypertensive drugs (kinds, IQR) & $2(1-3)$ & $3(1-4)$ & $3(2-4)$ & 0.074 \\
\hline $\operatorname{CCB}(n, \%)$ & $69(72.6 \%)$ & $55(72.4 \%)$ & $12(75.0 \%)$ & 0.428 \\
\hline $\operatorname{ACEI} / \operatorname{ARB}(n, \%)$ & $32(33.7 \%)^{*}$ & $18(23.7 \%)$ & $2(12.5 \%)$ & 0.029 \\
\hline$\beta$-blocker (n, \%) & $61(64.2 \%)^{*}$ & $39(51.3 \%)$ & $5(31.3 \%)$ & 0.033 \\
\hline Diuretic $(n, \%)$ & $23(24.2 \%)$ & $17(22.4 \%)$ & $6(37.5 \%)$ & 0.217 \\
\hline Clonidine $(n, \%)$ & $4(4.2 \%)^{*}$ & $5(6.6 \%)^{\&}$ & $4(25.0 \%)$ & 0.015 \\
\hline Revascularization operation ( $n, \%)$ & $28(29.5 \%)$ & $25(32.9 \%)$ & $5(31.3 \%)$ & 0.221 \\
\hline Events free survival by the end & $71.8 \% *$ & $65.8 \%$ & $50.0 \%$ & 0.041 \\
\hline
\end{tabular}

$A R$ aortic regurgitation; Cluster 1: involvement of abdominal aorta and/or renal artery; Cluster 2: involvement of ascending aorta, thoracic aorta, aortic arch, and its branches; Cluster 3: combined involvement of Cluster 1 and Cluster 2; CCB: calcium channel blocker; ACEI/ARB: angiotensin-converting enzyme inhibitor/ angiotensin receptor blocker; $p$ value: comparison among patients with different blood pressure control status; ${ }^{*} p<0.05$ for comparisons between patients with controlled and improved hypertension; ${ }^{*} p<0.05$ for comparisons between patients with controlled and failed controlled hypertension; ${ }^{\&} p<0.05$ for comparisons between patients with improved and failed controlled hypertension

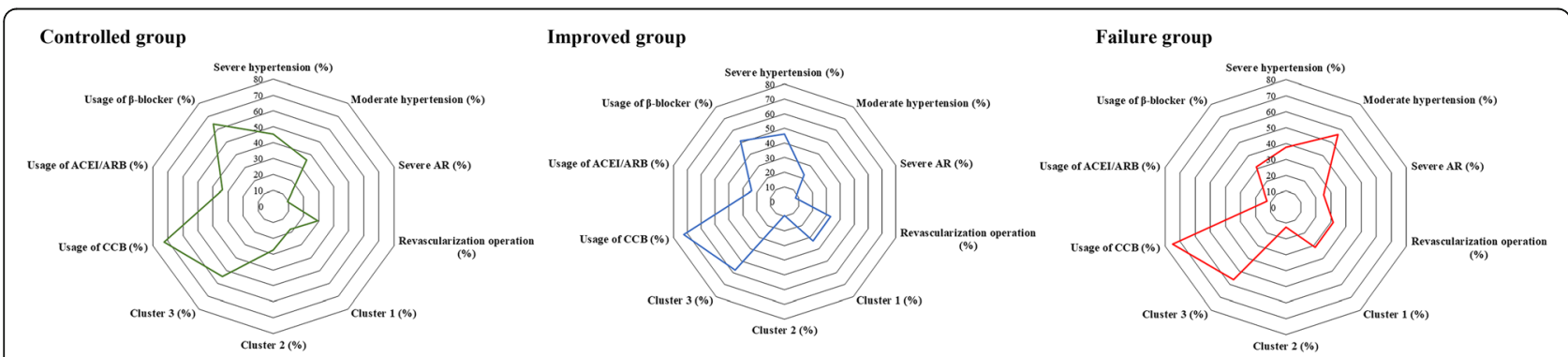

Fig. 3 Characteristics of patients with different blood pressure control status. Characteristics showed in the radar map included imaging phenotype (cluster), hypertensive degree, severe AR, and treatment (the usage of hypertensive drugs and Revascularization operation). AR: aortic regurgitation; CCB: calcium channel blocker; ACEI/ARB: angiotensin-converting enzyme inhibitor/angiotensin receptor blocker; Cluster 1: involvement of abdominal aorta and/or renal artery; Cluster 2: involvement of ascending aorta, thoracic aorta, aortic arch, and its branches; Cluster 3: combined involvement of Cluster 1 and Cluster 2 
Table 4 Multivariate Cox regression analysis of risk factors associated with events free survival during the follow-up

\begin{tabular}{|c|c|c|c|}
\hline & HR & $95 \% \mathrm{Cl}$ & $P$-value \\
\hline \multicolumn{4}{|l|}{ Baseline complications } \\
\hline Renal insufficiency & 0.84 & $0.77-3.18$ & 0.167 \\
\hline Heart dysfunction & 0.89 & $0.81-4.13$ & 0.241 \\
\hline Cerebrovascular events & 0.96 & $0.79-2.14$ & 0.383 \\
\hline Co-exist with severe AR & 0.87 & $0.64-0.95$ & 0.043 \\
\hline \multicolumn{4}{|l|}{ Imaging phenotype } \\
\hline Cluster 1 imaging phenotype & 0.69 & $0.48-0.92$ & 0.017 \\
\hline Cluster 2 imaging phenotype & 1.27 & $0.77-4.21$ & 0.441 \\
\hline Cluster 3 imaging phenotype & 0.72 & $0.43-0.94$ & 0.048 \\
\hline \multicolumn{4}{|l|}{ Baseline hypertensive severity } \\
\hline Mild & 1.79 & $0.78-1.32$ & 0.108 \\
\hline Moderate & 1.03 & $0.82-2.11$ & 0.094 \\
\hline Severe & 0.87 & $0.63-3.18$ & 0.069 \\
\hline \multicolumn{4}{|l|}{ Blood pressure control status } \\
\hline Controlled & 2.13 & $1.32-3.78$ & 0.047 \\
\hline Improved & 1.97 & $0.89-2.31$ & 0.136 \\
\hline Failure & 0.74 & $0.68-1.32$ & 0.087 \\
\hline Cluster $1+$ controlled blood pressure & 1 (reference) & & \\
\hline Cluster $1+$ uncontrolled blood pressure & 0.64 & $0.52-0.89$ & 0.031 \\
\hline Cluster 2+ controlled blood pressure & 2.21 & $1.47-4.32$ & 0.027 \\
\hline Cluster 2+ uncontrolled blood pressure & 1.48 & $0.89-3.11$ & 0.074 \\
\hline Cluster 3+ controlled blood pressure & 1.13 & $0.91-2.33$ & 0.069 \\
\hline Cluster 3+ uncontrolled blood pressure & 0.83 & $0.76-0.92$ & 0.048 \\
\hline
\end{tabular}

Cluster 1: involvement of abdominal aorta and/or renal artery; Cluster 2: involvement of ascending aorta, thoracic aorta, aortic arch, and its branches; Cluster 3: involvement of Cluster 1 plus Cluster 2; AR: aortic regurgitation; adjustment: age, sex, disease duration, as well as disease activity and treatment including medication and revascularization procedure; uncontrolled blood pressure: improved and failure blood pressure control status

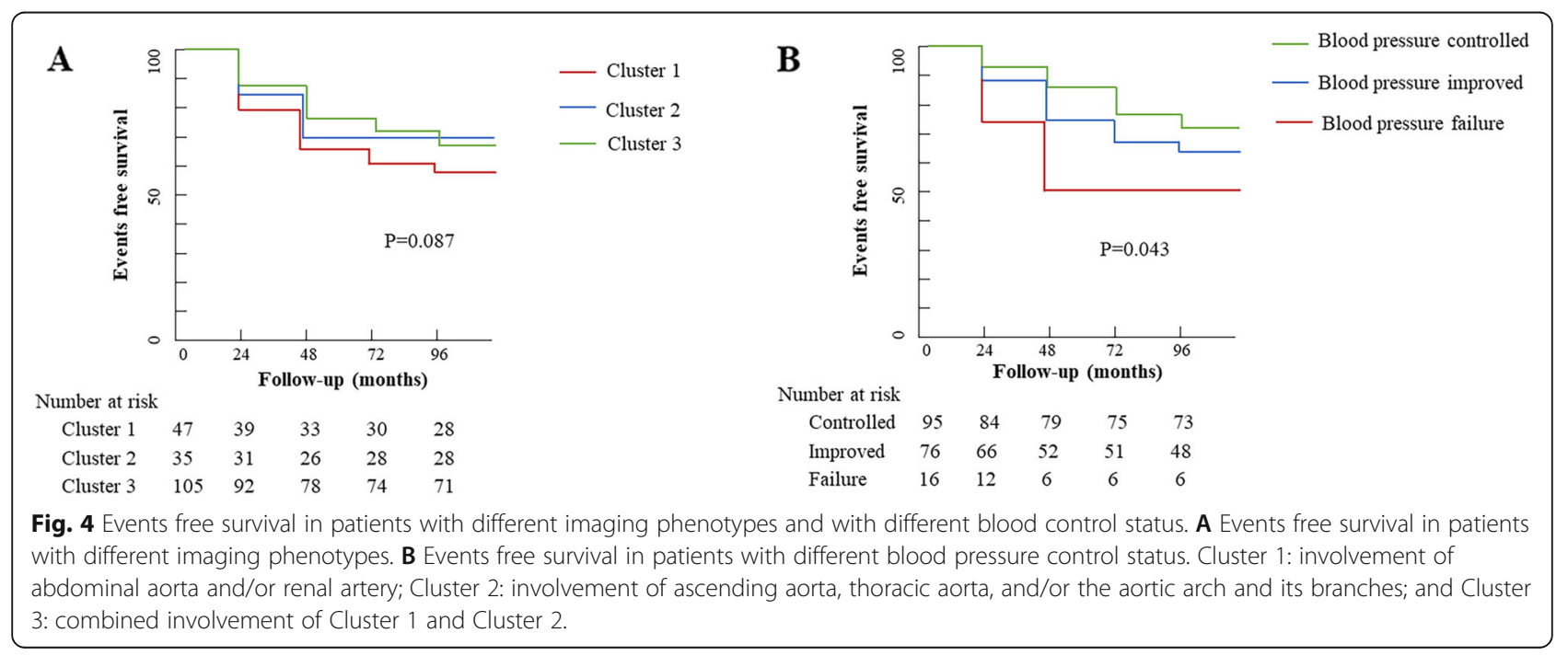


also found that the renal artery $(60 \%)$ was the most commonly involved artery in TAK patients with hypertension, and the prevalence of severe and refractory hypertension was significantly higher in patients with renal artery stenosis (data not shown), which might support the important role of renal artery stenosis in the causes of hypertension in TAK. In addition, significant differences of artery involvement were demonstrated between patients with and without hypertension, wherein it was speculated that hypertensive patients might have specific imaging phenotypes. We confirmed this by identifying three specific imaging phenotype clusters in patients with hypertension, which could be distinguished from cases without hypertension (Fig. 2). Younger age and worse disease status, especially the prevalence of severe hypertension and renal insufficiency, was observed in patients with Cluster 1 imaging phenotype. What is more, the imaging phenotypes defined in our study also showed significant effects on the EFS. The EFS was significantly lower in Cluster 1 (59.6\%) than that in Cluster 2, but similar to that in Cluster 3, which may be related to the higher prevalence of renal insufficiency and persistent refractory and/or malignant hypertension, as well as the lower prevalence of blood pressure control in Cluster 1 and Cluster 3. In addition, although renal and abdominal aorta involvement was indicated both in Cluster 1 and Cluster 3, Cluster 1 had a higher prevalence of severe $(>75 \%)$ renal artery stenosis than Cluster 3. Future studies would be needed to determine whether poor prognosis is mainly attributed to this involvement.

Except for renal artery, hypertension in TAK could be caused by multifactorial conditions. In Cluster 2, hypertension might be caused by the involvement of the ascending aorta, thoracic aorta, aortic arch, and its branches instead of the renal and abdominal aorta. Hamida et al. reported that lesions of supraaortic trunks, carotid lesions,

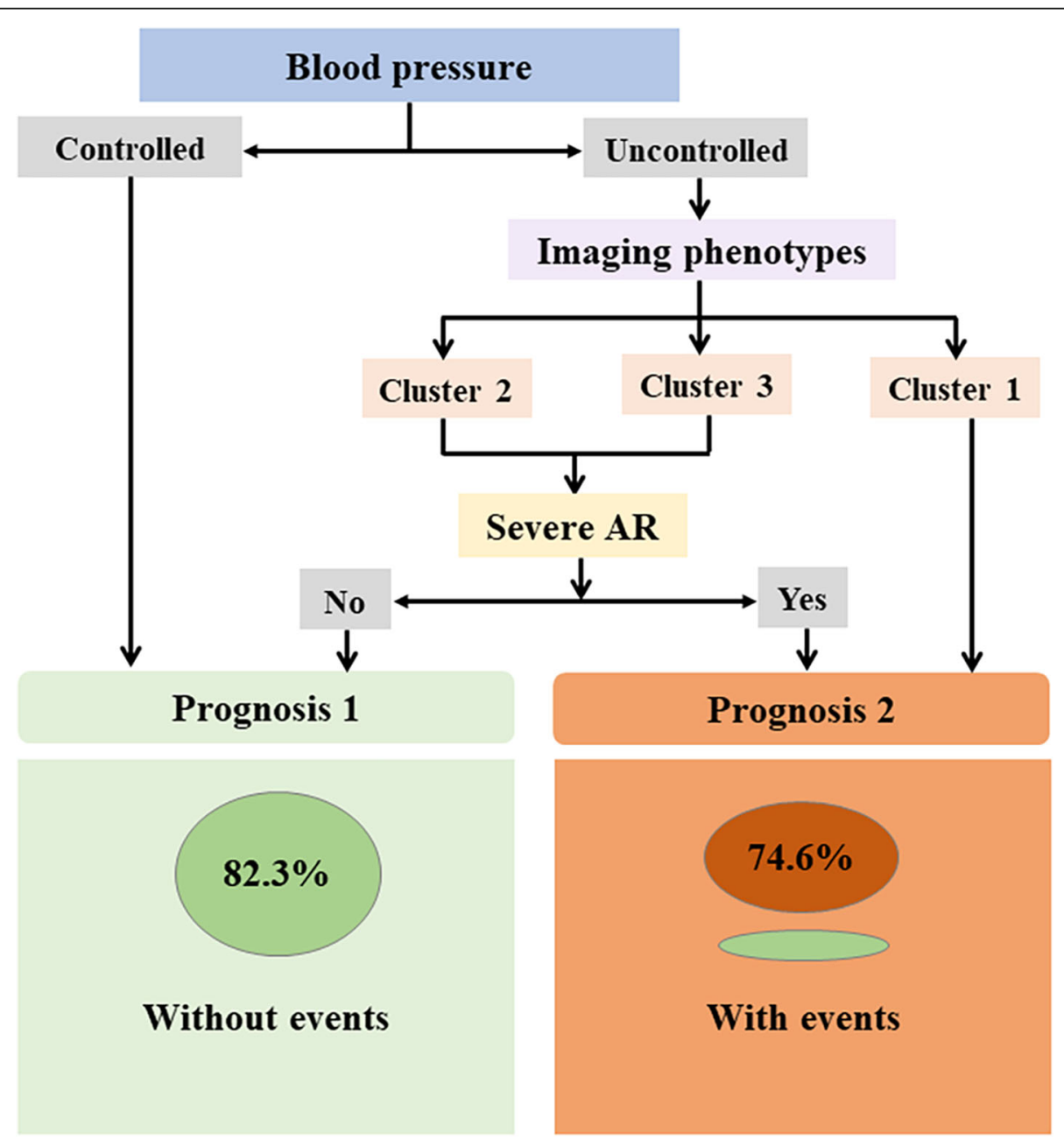

Fig. 5 Decision tree for predicting the prognosis of Takayasu arteritis patients with hypertension. Using three variables including imaging phenotype, blood pressure control status, and co-existence of severe AR, a decision tree diagram was established to predict the disease prognosis. Through the diagram, $69.2 \%$ of patients could be classified into the right prognosis group. AR: aortic regurgitation 
and immunosuppressive drugs might contribute to the genesis of hypertension in TAK [29]. Former studies have also found that dysfunctional baroreceptors are possible mechanisms involved in causing hypertension [30]. It is well recognized that a proatherogenic effect occurs in patients with TAK, which may increase arterial stiffness and decrease elasticity of arterial walls that may contribute to elevated blood pressure. In addition, severe AR was observed in $9.3 \%$ of hypertensive patients in our study, which was a little lower than that reported in a previous study [21]. Aortic regurgitation may be also associated with hypertension in TAK and is likely caused by directed valvular lesions, aneurysms arising from the aortic annulus, or annular dilation resulting from extensive dilatational changes of the ascending aorta. Furthermore, we also found that co-existence of severe AR was negatively related to the EFS. Thus, echocardiography monitoring is very necessary for the TAK population.

In the current investigation, only $50.8 \%$ of cases had blood pressure controlled during the follow-up, which was relatively low. More importantly, patients with blood pressure control showed significantly better EFS. Thus, the main treatment goal for TAK patients with hypertension should be not only to achieve and maintain disease remission, but also to achieve blood pressure control. For antihypertensive treatment, we found that patients with controlled blood pressure showed more usage of ACEI/ARB and $\beta$-blocker, which might indicate that ACEI/ARB and $\beta$ blocker could be a better choice for TAK patients with hypertension. However, in patients with bilateral renal artery involvement, ACEI/ARB was forbidden; in patients with unilateral renal artery involvement, close monitoring of serum creatinine and potassium should be done during the treatment with ACEI/ARB. Combined with the above data, we also made a decision tree diagram using three variables: imaging phenotype, blood pressure control status, and co-existence of severe AR (shown as Fig. 5). Through the diagram, $69.2 \%$ of patients could be classified into the right prognosis group. However, the power and accuracy of the decision tree diagram should be validated in the future, due to the small sample size of the present research.

Our study has two major limitations. First, due to the low incidence of TAK, association analyses between severity and controlled status as well as imaging phenotypic categories of hypertension with the prognosis may be underpowered, which warrants future larger studies to validate our results. Second, the follow-up duration was relatively short, and further studies with a larger sample size and longer follow-up duration are needed to validate the results.

\section{Conclusions}

In conclusion, 33\% of TAK patients suffered from hypertension in our cohort, with almost half severe cases.
Three specific imaging phenotypes were identified for TAK patients with hypertension. The blood pressure control rate was $50.8 \%$, with an overall EFS of $67.9 \%$ by the end of the follow-up. Our data support blood pressure control status and specific imaging phenotypes showed significant effects on EFS for hypertensive TAK patients.

\section{Abbreviations \\ TAK: Takayasu arteritis; AR: Aortic regurgitation; EFS: Events free survival; ECTA: East China Takayasu arteritis; ACR: American College of Rheumatology; ABI: Ankle brachial index; SBP: Systolic blood pressure; DBP: Diastolic blood pressure; MRA: Magnetic resonance angiography; CTA: Computed tomography angiography; GFR: Glomerular filtration rate; SD: Standard deviations; IQR: Interquartile range; HR: Hazard ratioCIConfidence interval; CCB: Calcium channel blocker; ACEI/ARB: Angiotensin-converting enzyme inhibitor/angiotensin receptor blocker; ESR: Erythrocyte sedimentation rate}

\section{Supplementary Information}

The online version contains supplementary material available at https://doi. org/10.1186/s13075-021-02579-8.

Additional file 1:. Fig S1. Tree dendogram for involved arteries of hypertensive and non-hypertensive Takayasu arteritis. Fourteen arteries including bilateral carotid arteries, brachiocephalic trunk, bilateral subclavian arteries, aortic arch, ascending aorta, thoracic aorta, pulmonary artery, abdominal aorta, bilateral renal artery, superior mesenteric artery and celiac axis were included in the cluster analysis by a two-step progress to identify imaging phenotypes for hypertensive population. Three specific imaging phenotype clusters was identified for hypertensive patients (A), which could be distinguished from non-hypertensive cases (B).

Additional file 2:. Supplementary Table 1. General characteristics in Takayasu arteritis patients with and without hypertension.

\section{Acknowledgements}

Not applicable.

\section{Authors' contributions}

Sun Ying: data analysis and paper written; Wu Sifan and Wang Yujiao: management of electronic database; Chen Rongyi, Huang Qingrong, Ma Lili, and Chen Huiyong: patients follow-up, clinical and imaging data collection; Jiang Lindi: study design. The authors read and approved the final manuscript.

\section{Funding}

This study was supported by National Natural Science Foundation of China (NSFC 81771730 and 81801598) and Clinical Cultivation Project of Shanghai Shenkang Science and Technology Development Center (SHDC12019X05).

Availability of data and materials

The datasets used and/or analyzed during the current study are available from the corresponding author on reasonable request.

\section{Declarations}

Ethics approval and consent to participate

The study was approved by the ethics committee of Zhongshan Hospital at Fudan University (B2013-115(3)).

Consent for publication

Informed written consent was obtained from each patient.

Competing interests

The authors declare that they have no competing interests. 
Received: 13 May 2021 Accepted: 9 July 2021

Published online: 21 July 2021

\section{References}

1. Johnston SL, Lock RJ, Gompels MM. Takayasu arteritis: a review. J Clin Pathol. 2002;55(7):481-6. https://doi.org/10.1136/jcp.55.7.481.

2. Watanabe $Y$, Miyata T, Tanemoto K. Current clinical features of new patients with Takayasu arteritis observed from a cross-country research in Japan: age and sex specificity. Circulation. 2015;132(18):1701-9. https://doi.org/10.1161/ CIRCULATIONAHA.114.012547.

3. Yilmaz N, Can M, Oner FA, Kalfa M, Emmungil $H$, Karadag $O$, et al. Impaired quality of life, disability and mental health in Takayasu's arteritis. Rheumatology (Oxford). 2013:52:1898-904.

4. Laurent A, Julien H, Nicolas L, Toledano D, Gambotti L, Chalumeau NC, et al. Takayasu arteritis in France: a single-center retrospective study of 82 cases comparing white, North African, and black patients. Medicine. 2010;89:1-17.

5. Mwipatayi BP, Jeffery PC, Beningfield SJ, Matley PJ, Naidoo NG, Kalla AA, et al. Takayasu arteritis: clinical features and management: report of 272 cases. ANZ J Surg. 2005;75(3):110-7. https://doi.org/10.1111/j.1445-2197.2005. 03312.x.

6. Park MC, Lee SW, Park YB, Chung NS, Kee SK. Clinical characteristics and outcomes of Takayasu's arteritis: analysis of 108 patients using standardized criteria for diagnosis, activity assessment, and angiographic classification. Scand J Rheumatol. 2005;34(4):284-92. https://doi.org/10.1080/0300974051 0026526.

7. Jain S, Kumari S, Ganguly NK, Sharma BK. Current status of Takayasu arteritis in India. Int J Cardiol. 1999;54:S111-6.

8. Park YB, Hong SK, Choi KJ, Sohn DW, Oh BH, Lee MM, et al. Takayasu arteritis in Korea: clinical and angiographic features. Heart Vessel. 1992;7:559.

9. Zheng D, Fan D, Liu L. Takayasu arteritis in China: a report of 530 cases. Heart Vessel. 1992;7:32-6.

10. Mammeri A, Guermaz R, Hatri A, Kessal F, Taharboucht S, Hamrour F, et al. Hypertension during Takayasu's disease: an Algerian single center experience of 279 patients. Annales de cardiologie et d'angeiologie. 2017; 66(3):154-8. https://doi.org/10.1016/j.ancard.2017.04.006.

11. Maksimowicz-Mckinnon K, Clark TM, Hoffman GS. Limitations of therapy and a guarded prognosis in an American cohort of Takayasu arteritis patients. Arthritis Rheum. 2007:56(3):1000-9. https://doi.org/10.1002/art.22404.

12. Sato El, Lima D, Santo BE, Hata F. Takayasu arteritis. Treatment and prognosis in a university center in Brazil. Int J Cardiol. 2000;75:S163-6. https://doi.org/10.1016/S0167-5273(00)00197-2.

13. Hong S, Ghang B, Kim YG, Lee CK, Yoo B. Long term outcomes of renal artery involvement in Takayasu arteritis. J Rheumatol. 2017:44(4):466-72. https://doi.org/10.3899/jrheum.160974.

14. Sun Y, Dai X, Lv P, Dong Z, Ma L, Yan Y, et al. Characteristics and mediumterm outcomes of Takayasu arteritis-related renal artery stenosis: analysis of a large Chinese cohort. J Rheumatol. 2021;48(1):87-93. https://doi.org/10.3 899/jrheum.190965.

15. Petrovic-Rackov L, Pejnovic N, Jevtic M, Damjanov N. Longitudinal study of 16 patients with Takayasu's arteritis: clinical features and therapeutic management. Clin Rheumatol. 2009;28(2):179-85. https://doi.org/10.1007/s1 0067-008-1009-7.

16. Kaoru M, Hitoshi O, Junjiro K, Ishibashi-Ueda H, Matsuda H, Minatoya K, et al. Surgical treatment of aortic regurgitation due to Takayasu arteritis: longterm morbidity and mortality. Circulation. 2005;112:3707-12.

17. VanoliM DE, Salvarani C, Rossi C, Bacchiani G, Schieppati A, et al. Takayasu's arteritis: a study of 104 Italian patients. Arthritis Rheum. 2005;53(1):100-7. https://doi.org/10.1002/art.20922.

18. Arend WP, Michel BA, Bloch DA, Hunder GG, Calabrese LH, Edworthy SM, et al. The American College of Rheumatology 1990 criteria for the classification of Takayasu arteritis. Arthritis Rheum. 1990;33(8):1129-34. https://doi.org/10.1002/art.1780330811.

19. Kerr FS, Hallahan CW, Gordano J, Leavitt RY, Fauci AS, Rottem M, et al. Takayasu arteritis. Ann Intern Med. 1994;120(11):919-29. https://doi.org/10. 7326/0003-4819-120-11-199406010-00004.

20. Cifkova R, Erdine S, Fagard R, Farsang C, Heagerty AM, Kiowski W, et al. Practice guidelines for primary care physicians: 2003 ESH/ESC hypertension guidelines. J Hypertens. 2003:21(10):1779-86. https://doi.org/10.1097/01.hjh. $0000084773.37215 .1 \mathrm{~b}$.
21. Qi Y, Yang $L$, Zhan $H$, Liang E, Song $L$, Cai J, et al. The presentation and management of hypertension in a large cohort of Takayasu arteritis. Clin Rheumatol. 2018;37(10):2781-8. https://doi.org/10.1007/s10067-017-3947-4.

22. Hata A, Noda M, Moriwaki R, Numano F. Angiographic findings of Takayasu arteritis: new classification. Int J Cardiol. 1996;54:S155-63. https://doi.org/10.1 016/S0167-5273(96)02813-6.

23. Bonow RO, Carabello B, de Leon AC, Edmunds LH, Fedderly BJ, Freed MD, et al. ACC/AHA guidelines for the management of patients with valvular heart disease. Executive summary. A report of the American College of Cardiology/American Heart Association Task Force on practice guidelines (Committee on management of patients with valvular heart disease). J Heart Valve Dis. 1998;7(6):672-707.

24. Bekeredjian R, Grayburn PA. Valvular heart disease: aortic regurgitation. Circulation. 2005;112(1):125-34. https://doi.org/10.1161/CIRCULATIONAHA.1 04.488825.

25. Zoghbi WA, Enriquez-Sarano M, Foster E, Grayburn PA, Kraft CD, Levine RA, et al. Recommendations for evaluation of the severity of native valvular regurgitation with two-dimensional and Doppler echocardiography. J Am Soc Echocardiogr. 2003;16(7):777-802. https://doi.org/10.1016/S0894-7317(03 )00335-3.

26. Grayson PC, Maksimowicz-McKinnon K, Clark TM, Tomasson G, Cuthbertson D, Carette $S$, et al. Distribution of arterial lesions in Takayasu's arteritis and giant cell arteritis. Ann Rheum Dis. 2012;71(8):1329-34. https://doi.org/10.113 6/annrheumdis-2011-200795.

27. Evan F, Daniel A, Terri F. Hypertension and elevated ESR as diagnostic features of Takayasu arteritis in children. J Clin Rheumatol. 2003;9:156-63.

28. Peng $M$, Jiang XJ, Dong H, Zou YB, Zhang HM, Song L, et al. Etiology of renal artery stenosis in 2047 patients: a single-center retrospective analysis during a 15-year period in China. J Hum Hypertens. 2016;30(2):124-8. https:// doi.org/10.1038/jhh.2015.40.

29. Hmida B, Maazoun M, et al. Hypertension in Takayasu disease: Pp.15.72. J Hypertens. 2010;28:e270. https://doi.org/10.1097/01.hjh.0000378998.52257.6d.

30. Milner LS, JacobsDW TPD, Kala UK, Franklin J, Beale P, et al. Management of severe hypertension in childhood Takayasu's arteritis. Pediatr Nephrol. 1991; 5(1):38-41. https://doi.org/10.1007/BF00852840.

\section{Publisher's Note}

Springer Nature remains neutral with regard to jurisdictional claims in published maps and institutional affiliations.

Ready to submit your research? Choose BMC and benefit from:

- fast, convenient online submission

- thorough peer review by experienced researchers in your field

- rapid publication on acceptance

- support for research data, including large and complex data types

- gold Open Access which fosters wider collaboration and increased citations

- maximum visibility for your research: over $100 \mathrm{M}$ website views per year

At BMC, research is always in progress.

Learn more biomedcentral.com/submissions 